\title{
Salt-related structure geologic model of Pre-Caspian Basin and analysis on imaging capability
}

\author{
Yaojun Wang' Yanyan $\mathrm{Ma}^{2} \cdot$ Guofa $\mathrm{Li}^{3} \cdot \mathrm{Hao} \mathrm{Li}^{4} \cdot$ \\ Naxin $\operatorname{Tian}^{5} \cdot$ Yongfu Cui ${ }^{6}$
}

Received: 10 April 2015/Accepted: 22 January 2016/Published online: 6 February 2016

(C) Akadémiai Kiadó 2016

\begin{abstract}
The Pre-Caspian Basin is one of the most prolific petroliferous basins in Kazakhstan. However, the hydrocarbon reservoir is always located in salt dome and presalt structures. The salt-related structure is so complex that it is difficult to obtain the satisfied imaging results. In order to improve the imaging precision and provide authentic amplitude information to reservoir prediction, we should analyze the imaging and amplitude preserving capability between different migration methods. According to actual geologic and reservoir characteristics, we design a typical complex salt-related structure model of the basin. Based on the forward modelling seismic record, we compare the one-
\end{abstract}

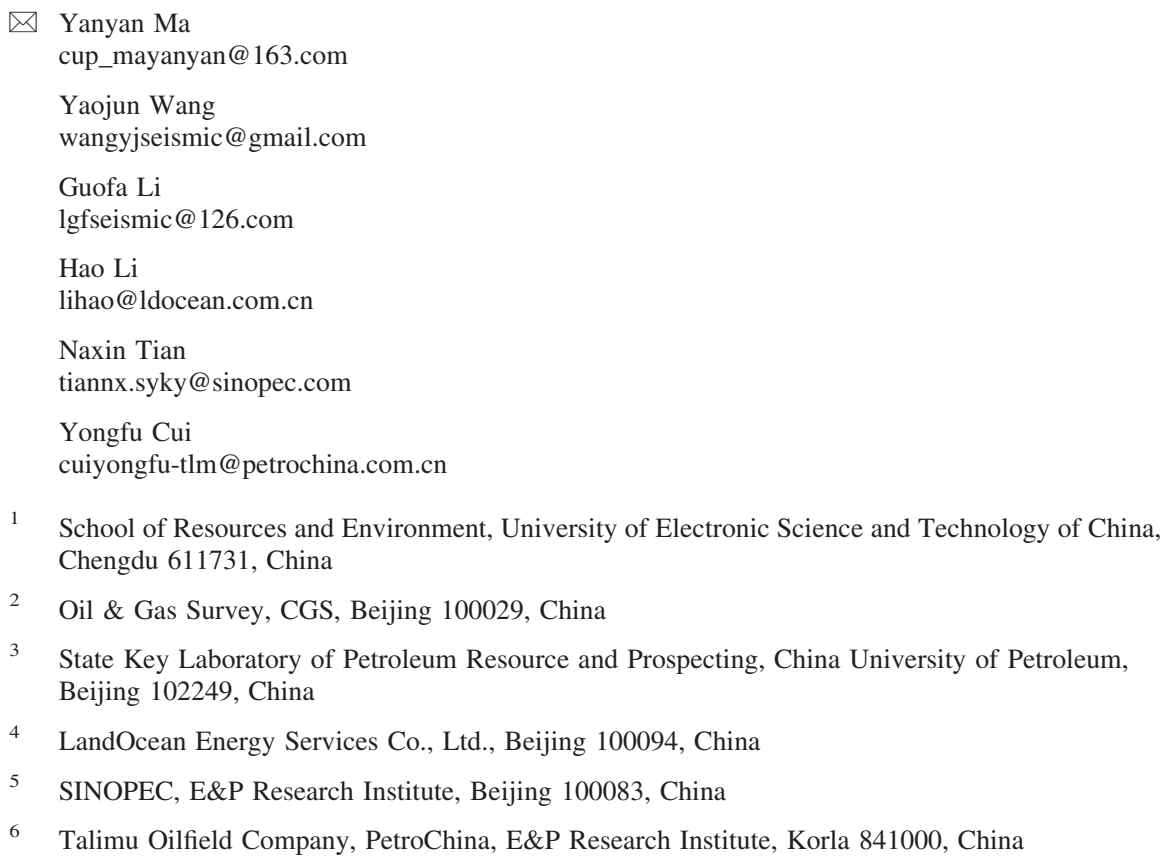


way wave-equation pre-stack depth migration (WPDM) and pre-stack reverse-time depth migration (RTM). The results show that RTM can greatly improve the imaging quality of salt dome, but its improvement for the pre-salt structures is small compared with WPDM. Both methods are equally capable of preserving seismic reflection characteristics that affected mainly by the lateral variation of overlying strata.

Keywords Pre-Caspian Basin - Salt-related structure geologic model · RTM · WPDM · Imaging capability

\section{Introduction}

The Pre-Caspian Basin is a typical salt basin, where more than 1800 salt structures have been found (Volozh et al. 2003). Recently, with the unceasing development of exploration, the target turns to deep pre-salt reservoirs. However, since the distinct velocity contrast between the overlying thick salt domes and surrounding rocks, the pre-salt structures are difficult to image. Therefore, it is urgent to adopt precisely migration algorithm to image the salt-related structures in Pre-Caspian Basin.

Because the salt dome with the large variable lateral velocity, it is better to utilize the pre-stack depth migration methods to obtain the more precisely imaging results. As we know, there has several depth migration methods been used in subsurface imaging. Depending on their underlying assumptions, the migration methods can be classified as either Kirchhoff (Sun et al. 2000; Schneider 1978) or wavefield extrapolation (Gazdag 1978; Bleistein 1987; Stolt 1978) methods. The Kirchhoff method is a high-frequency approximation of the wave equation, so that it may not provide reliable subsurface images in the complex geological area where multi-pathing occurs (Leveille et al. 2011). In wavefield extrapolation methods, the multi-pathing is handled in a natural way. Especially for the salt dome imaging, the wave-field methods are better than Kirchhoff methods (Leveille et al. 2011). There are two types of the wavefield extrapolation methods, one-way and two-way methods. The one-way wave-equation pre-stack depth migration (WPDM) adopts wave field continuation for imaging and can get rid of the ray tracing limitations, but it fails to process the reverse branch generated at the flanks of salt (Gazdag 1978). The two-way wave-equation methods, such as pre-stack reverse-time migration (RTM) makes the upward and downward waves focus onto the exact reflection position which provide an accurate description of wave propagation, so it has a lot of successful applications in the high-steep and salt-related structures imaging (Baysal et al. 1983; Willis et al. 2006).

In theory, the superiority of RTM method on salt dome imaging is more than WPDM, which has been introduced in a large number of literatures (Leveille et al. 2011; Zhang et al. 2007a, b; Etgen et al. 2009). Naturally, RTM is the first choice to improve the imaging quality of the salt dome structures in Pre-Caspian Basin. However, the verification of RTM is using the salt dome model of the Gulf of Mexico deep-water oil field (Liu et al. 2009; Zhang et al. 2007a, b; Liu et al. 2012). It is necessary to analyze and compare the imaging capabilities of RTM and WPDM based on the actual regional geologic characteristics, and then give some suggestions to apply the migration method to the real seismic data processing.

In this paper, we establish the 2D typical complex salt-related structure geologic model according to the actual geologic structure in Pre-Caspian Basin, and then we simulate the 
wave-field of acoustic wave. After that, we compare the imaging and amplitude preserving capabilities of WPDM and RTM methods using the exactly velocity model. At last, in order to analyze the influence of the initial velocity model, we also compare the imaging and amplitude preserving capabilities of WPDM and RTM methods by using the erroneous velocity model calculated from the real seismic processing. Finally, based on these analysis results, some conclusions and suggestions for migration imaging in Pre-Caspian Basin are drawn.

\section{Stratigraphic characteristics of Pre-Caspian Basin}

As a typical salt-bearing basin, the Pre-Caspian Basin is regarded as the one of the prospective areas in Kazakhstan. The sediments in the basin can be divided into formations, marked separately by the gypsiferous salt sedimentary in the Kungurian of Lower Permian: pre-salt, salt and post-salt formation. Present explorations demonstrate that the post-salt is dominated by clastic sedimentary characterized by large numbers of shallow burial with scattered reserve of small scale. However, the reservoirs in the pre-salt formation, mostly related to the organic reefs, are characterized by few quantities but large scale of splendid reserve. It indicates the pre-salt formation has great prospecting potential of hydrocarbon exploration. As the geological structure of the salt dome is complex, there is a great challenge for the basin imaging. Figure 1 shows a real seismic profile for the basin after Kirchhoff pre-stack depth migration. The boundary of salt structure is fuzzy and the pre-salt formation is scattered, which make the structures identification and reservoir prediction become difficult. Therefore, it is of great significance to adopt the useful algorithm to image the structure of basin precisely, and at the same time preserve the amplitude information as much as possible. The wavefield extrapolation methods, WPDM and RTM are the appropriate choices. In order to determine the imaging method reasonable, we establish a salt-related structure geologic model according to the actual stratigraphic characteristics of Pre-Caspian Basin, and then we make the specific analysis on imaging and amplitude-preserving capability of WPDM and RTM methods.

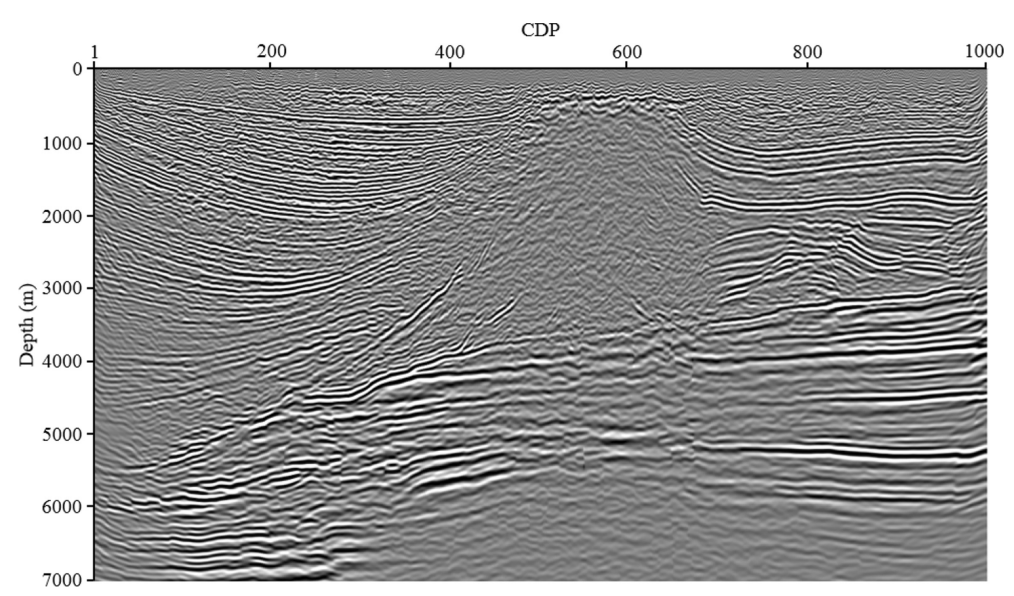

Fig. 1 Typical seismic profile of Pre-Caspian Basin after Kirchhoff pre-stack depth migration 


\section{Typical salt-related structure model and wave field simulation}

Based on the geologic characteristics, seismic features and well-logging data, the 2D typical complex salt-related structure geologic model of Pre-Caspian Basin is established. The result is displaying in Fig. 2a. The maximum depth of model is $7000 \mathrm{~m}$ and the length along horizontal direction is $1200 \mathrm{~m}$. According to stratigraphic characteristics, three sedimentary series are simulating from the top to down: the post-salt, salt and pre-salt strata. The post-salt strata simulate clastic-dominated deposition and are almost flat as a whole. However, the salt-related tectonic movements cause complex faulting systems and fragmentized formations. We design three salt domes with different scale and shape, from left to right, they are marked as Salt Domes 1-3. The maximum dip angle along the both sides of Salt Dome 1 and the left side of Salt Dome 2 is about $45^{\circ}$, while, it is about $75^{\circ}$ along the right side of Salt Dome 2 and both sides of Salt Dome 3. The pre-salt strata, the research emphasis of this paper, are buried deep and simulate marine carbonate deposition
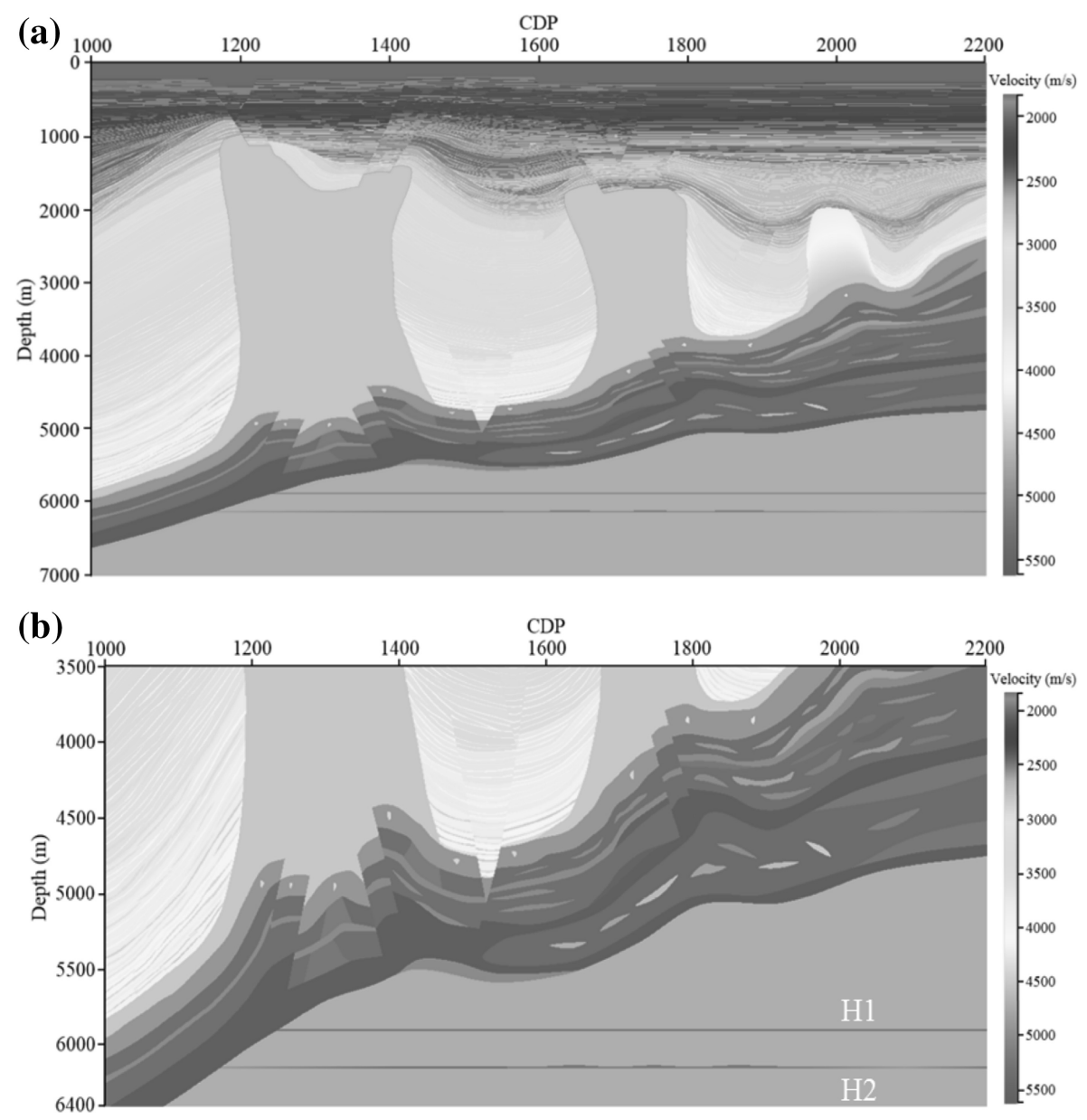

Fig. 2 The typical complex salt-related structure model (a) and the fractionated gain of pre-salt strata (b) 
with organic reef and oolitic beach. Figure $2 \mathrm{~b}$ displays the fractionated gain of pre-salt strata. In addition, two fictitious horizontal thin layers, $\mathrm{H} 1$ and $\mathrm{H} 2$, are designed at depth of 6000 and $6150 \mathrm{~m}$ in Fig. 2b, to test the amplitude-preserving performance of WPDM and RTM methods. The thickness and velocity of $\mathrm{H} 1$ remain unchanged in lateral. The thickness and velocity of $\mathrm{H} 2$ divide into four parts. In the first part, thickness keeps stable but velocity increases linearly from left to right, in the second part, both thickness and velocity stay stable, in the third part, velocity keeps stable but thickness changes periodically, in the last part, thickness keeps stable but velocity firstly increases and then decreases linearly.

Since the present migration methods are generally based on acoustic wave equation, to avoid the influence of wave field separation in elastic wave simulation, we adopt the firstorder velocity-pressure acoustic equation (Virieux 1984) to simulate the pre-stack wavefield,

$$
\left\{\begin{array}{c}
\frac{\partial p}{\partial t}=-K\left(\frac{\partial v_{x}}{\partial x}+\frac{\partial v_{z}}{\partial z}\right)+f(t) \delta\left(\mathbf{x}-\mathbf{x}_{o}\right), \\
\frac{\partial v_{x}}{\partial t}=-\frac{1}{\rho} \frac{\partial p}{\partial x} \\
\frac{\partial v_{z}}{\partial t}=-\frac{1}{\rho} \frac{\partial p}{\partial z}
\end{array}\right.
$$

where $p$ is the pressure wave field, $\rho$ is the medium density, $x$ is the horizontal coordinate, $z$ is the vertical coordinate, $v_{x}$ and $v_{z}$ are the two components of particle velocity along horizontal and vertical directions, respectively, $f(t)$ is the source time series, $\delta$ is the unit impulse response function, $\mathbf{x}_{o}$ is the source location, and $K$ is the elasticity modulus which is expressed as

$$
K=\rho v^{2} .
$$

A perfectly matched layer is used for the artificial boundary to eliminate the boundary reflection (Yuan et al. 2014). Meanwhile, to remove the effect of boundary reflection completely and ensure the folds of the entire model, both sides of the model are expanded to 1000 CDPs $(2000 \mathrm{~m})$. Mid-point shot is used in the simulation to give a total of 540 shot records. The 2D geometry parameters are as following: the source is Ricker wavelet with $30 \mathrm{~Hz}$ central frequency, the shot interval is $40 \mathrm{~m}$, the offset range is from 0 to $4000 \mathrm{~m}$ and the offset interval is $20 \mathrm{~m}$, the time record is $6 \mathrm{~s}$ and the sample interval is $2 \mathrm{~ms}$. Figure 3 is the shot record, when the source is locating at CDP 1600 in Fig. 2a. From this record, we can see intricate wave fields, involving direct wave, reflection wave, diffraction wave and other waves. Ignoring background noise, the synthetic record has a high similarity with the real seismic data acquired from Pre-Caspian Basin. It implies that our forward modelling appropriate for the real situation.

\section{Imaging capability comparison}

Because the velocity model may affect the imaging results, we firstly test the imaging capability of WPDM and RTM by using the exact velocity model (Fig. 2a). The WPDM method is utilized to image the model. We can decompose Eq. (1) to the upward wave Eq. (3) and downward wave Eq. (4) (Zhang et al. 2007a, b), 


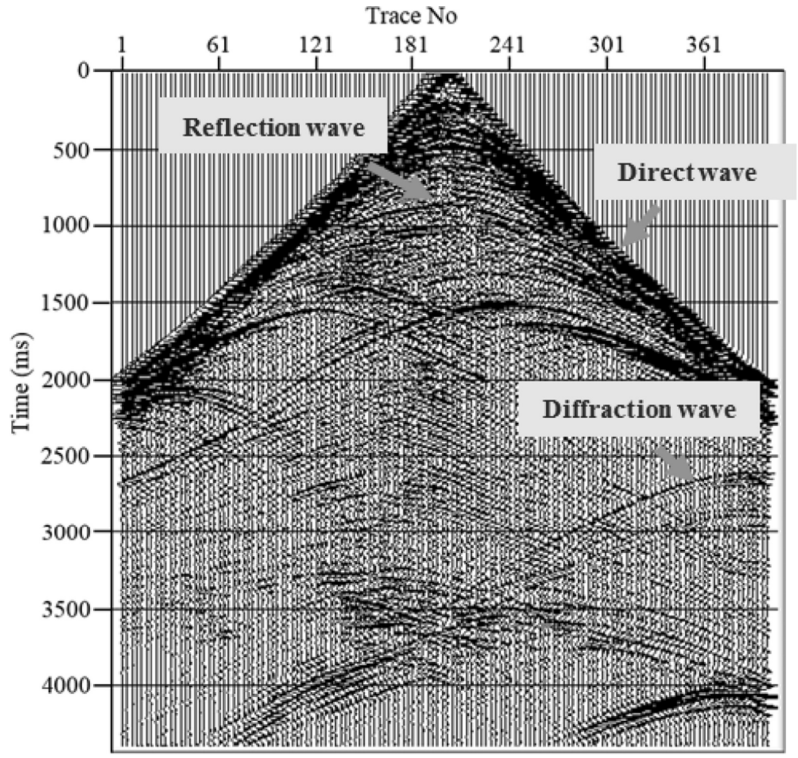

Fig. 3 The synthetic single shot record

$$
\begin{aligned}
& \left\{\begin{array}{c}
\left(\frac{\partial}{\partial z}-i \Lambda-\Gamma\right) p_{U}\left(\mathrm{X} ; \mathrm{X}_{s} ; \omega\right)=0, \\
p_{U}\left(x, 0 ; x_{s}, 0 ; \omega\right)=u\left(x ; x_{s} ; \omega\right),
\end{array}\right. \\
& \left\{\begin{array}{c}
\left(\frac{\partial}{\partial z}+i \Lambda-\Gamma\right) p_{D}\left(\mathrm{X} ; \mathrm{X}_{s} ; \omega\right)=0, \\
p_{D}\left(x, 0 ; x_{s}, 0 ; \omega\right)=\delta\left(x-x_{s}\right) .
\end{array}\right.
\end{aligned}
$$

In which, $\mathrm{X}=(x, z), \Lambda$ and $\Gamma$ represent pseudo-differential operators given by

$$
\begin{gathered}
i \Lambda=\frac{i \omega}{v} \sqrt{1-\frac{\left(v \partial_{x}\right)^{2}}{\omega^{2}}}, \\
\Gamma=\frac{\partial_{z} v}{2 v}\left[1+\frac{\left(v \partial_{x}\right)^{2}}{\omega^{2}-\left(v \partial_{x}\right)^{2}}\right] .
\end{gathered}
$$

In Eqs. (5) and (6), $\mathrm{X}_{s}=\left(x_{s}, 0\right)$ is the shot location, $p_{D}$ is the downgoing response to the impulsive boundary condition at $\mathrm{z}=0$, and $p_{U}$ is the upgoing wave that equal the observed seismic data $u\left(x ; x_{s} ; \omega\right)$ at the upper surface. If we extrapolate the upgoing wave along the negative direction of $\mathrm{z}$ axis using Eq. (3), we can get the upward wave-field at each depth. Similarly, giving the source wavelet and extrapolating it along $\mathrm{z}$ axis using Eq. (4), we can get the wave-field of downgoing wave. At last, the following correlationtype imaging condition (Zhang et al. 2007a, b) is utilized to obtain the image:

$$
I\left(\mathrm{X} ; x_{s}\right)=\int-i \times \operatorname{sgn}(\omega) p_{U}\left(\mathrm{X} ; \mathrm{X}_{s} ; \omega\right) p_{D}^{*}\left(\mathrm{X} ; \mathrm{X}_{s} ; \omega\right) d \omega .
$$


Here, the multiplier $-i \times \operatorname{sgn}(\omega)$ is introduced to obtain the correct inversion phase in 2D. $p_{U} p_{D}^{*}$ is the correlation product of waveforms.

After that, we implement RTM to migration. Different from WPDM, RTM method adopts two-way wave Eqs. (8) and (9) to compute the source wavefield $P_{F}$ and recorded receiver wavefield $P_{B}$, respectively.

$$
\begin{gathered}
\left\{\begin{array}{c}
\left(\frac{1}{v^{2}} \frac{\partial^{2}}{\partial t^{2}}-\nabla^{2}\right) p_{F}\left(x ; x_{s} ; t\right)=0 \\
p_{F}(x, z=0 ; t)=\delta\left(x-x_{s}\right) \int f\left(t^{\prime}\right) d t^{\prime}
\end{array}\right. \\
\left\{\begin{array}{l}
\left(\frac{1}{v^{2}} \frac{\partial^{2}}{\partial t^{2}}-\nabla^{2}\right) p_{B}\left(x ; x_{s} ; t\right)=0, \\
p_{B}(x, z=0 ; t)=u\left(x, x_{s} ; t\right),
\end{array}\right.
\end{gathered}
$$

where $v$ is the velocity, $f(t)$ is the source signature, and $\nabla^{2}$ is the Laplacian operator. At last, the similar correlation-type imaging condition is employed to obtain the image (Zhang et al. 2014),

$$
I\left(\mathrm{X} ; x_{s}\right)=\int p_{B}\left(\mathrm{X} ; \mathrm{X}_{s} ; t\right) p_{F}^{*}\left(\mathrm{X} ; \mathrm{X}_{s} ; t\right) d t .
$$

The imaged results of WPDM and RTM methods are shown in Fig. 4a, b, respectively.

Compared with the geologic model in Fig. 2a and the migrated record in Fig. 4, we can observe that both two methods successfully recover the sedimentary structures. However, there are still differences in detail. Figure 4a shows WPDM can depict the salt dome shape roughly but fail to image salt flank and high dip formations along the right side of Salt Dome 2. On the contrary, for the salt dome, the flank and stratigraphic contact feature is more clearly at the migrated profile using RTM, which can be seen at the marked by arrows in Fig. 4b. Therefore, for the salt dome, the comparison shows that RTM has the better effect than WPDM. For pre-salt strata, although RTM image the faulting system more obviously, which is marked by white ellipse in Fig. 4, the enhancement of pre-salt strata is not obviously. Here, it should be noted that although the imaging of salt dome flank cannot affect the imaging of pre-salt structures directly, the confirmation of the flank does influence the accuracy of velocity modeling and then affect the imaging quality of pre-salt structures.

In practical, geologists always interest in the favorable reservoir at upper of the structure. Therefore, imaging depth error becomes the important index to evaluate the migration methods. Here, we calculate the reflection coefficient from the exact velocity model (Fig. 2a), and then utilize the $30 \mathrm{~Hz}$ Ricker wavelet to make the convolution operation. Figure 5 is the synthetic record in depth domain after making time-depth conversion. The convolution record accurately reflects the structure and reservoir characteristic in post-stack profile. We take this convolution model as a reference to analyze the depth error of WPDM and RTM methods quantificationally.

We pick the seismic event marked by arrows in Figs. 4 and 5, respectively. Figure 6 is the depth error curves of two methods, which are calculated by taking the depth of the same event in convolution record as a reference. We can see that the overall error of RTM is slightly smaller than that of WPDM, but the difference between the two error curves hardly exists for the formations about $4000 \mathrm{~m}$ underground. It demonstrates that for presalt strata, the imaging capability of RTM does not have obvious superiority over WPDM. This is because when seismic wave propagates through the salt strata, one-way wave 

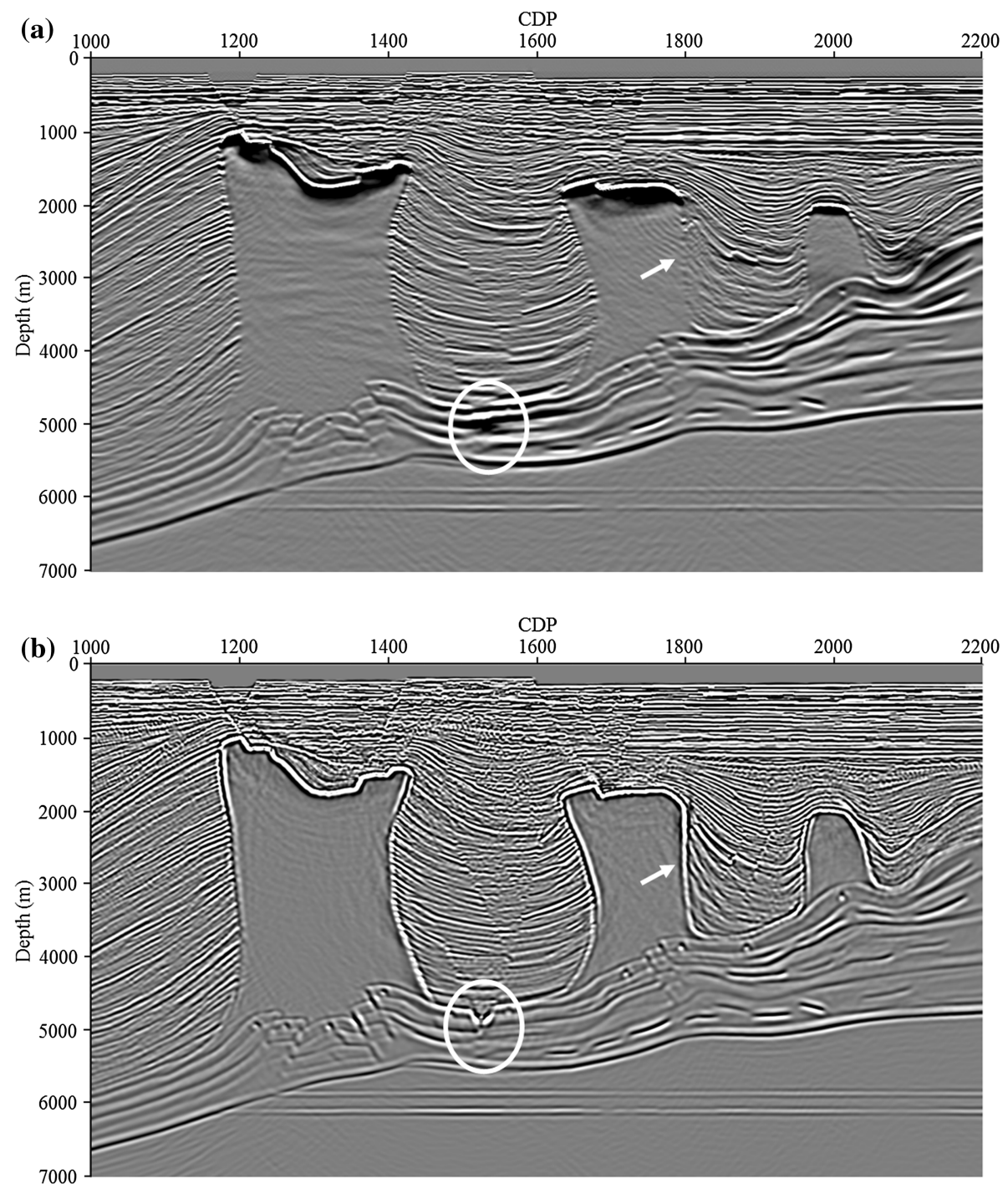

Fig. 4 Comparison between migration profiles of WPDM (a) and RTM (b) using the exact velocity model

equation fails to handle reverse branch generated at the salt dome, whereas two-way wave equation is free of dip angle restriction, can better image the reverse branch. Therefore, RTM has a significant advantage over WPDM when it comes to salt dome imaging. However, reverse branch is not generated in seismic wave propagation through this pre-salt flat strata, meaning in this case RTM loses its intrinsic advantage and is not inherently better than WPDM.

After migrating the seismic data, it is always required to acquire the information of reservoir lithology and fluid distribution by seismic inversion and attribute analysis. The reliability of these methods largely depends on the amplitude fidelity of seismic data processing. Therefore, the amplitude-preserving capability is also the important index to 


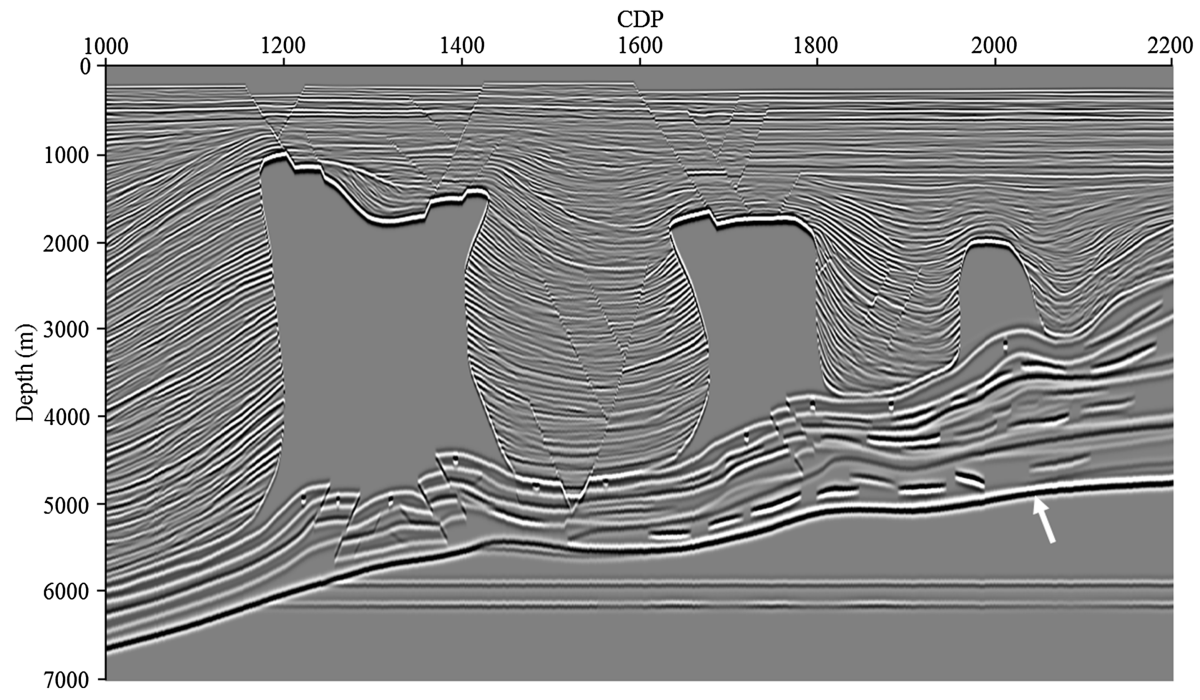

Fig. 5 The convolution record in the depth domain

Fig. 6 Comparison between imaging depth errors of WPDM result (dash curve) and RTM result (solid curve) at the same event marked by arrow in Figs. 4 and 5

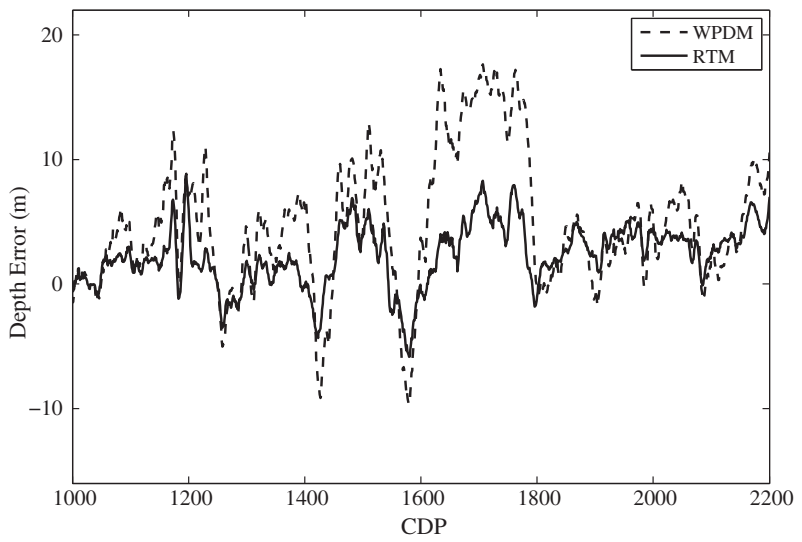

evaluate the migration methods. Analogously, taking the convolution record as reference, we analyze the amplitude-preserving capability of the two methods using the amplitude information of horizontal layers $\mathrm{H} 1$ and $\mathrm{H} 2$ (Fig. 2). Because the thickness and velocity of $\mathrm{H} 1$ remain unchanged in lateral, its reflection amplitude should also maintain invariant if we ignore the effect of overlying strata. The second layer $\mathrm{H} 2$ varies in both thickness and velocity, and thus its reflection amplitude should change laterally as a result. This is an important clue for identification of reservoir properties in seismic data interpretation. Figure 7 illustrates the amplitude variation curves for $\mathrm{H} 1$ and $\mathrm{H} 2$, which are picked from the WPDM result (Fig. 4a), RTM result (Fig. 4b) and convolution record (Fig. 5), respectively. The amplitude curves of convolution record can accurately reveal the thickness and velocity variations, but those of the two methods differ greatly from that of convolution record. In particular, amplitude curve of convolution record at H1 layer is a 
Fig. 7 Comparison between amplitude variation curves of convolution record (dash-dot curve), WPDM (solid curve) and RTM (dash curve) results using the true velocity model. a Curves at the $\mathrm{H} 1$ around $6000 \mathrm{~m}$, and b curves at $\mathrm{H} 2$ around $6150 \mathrm{~m}$ in Fig. 2
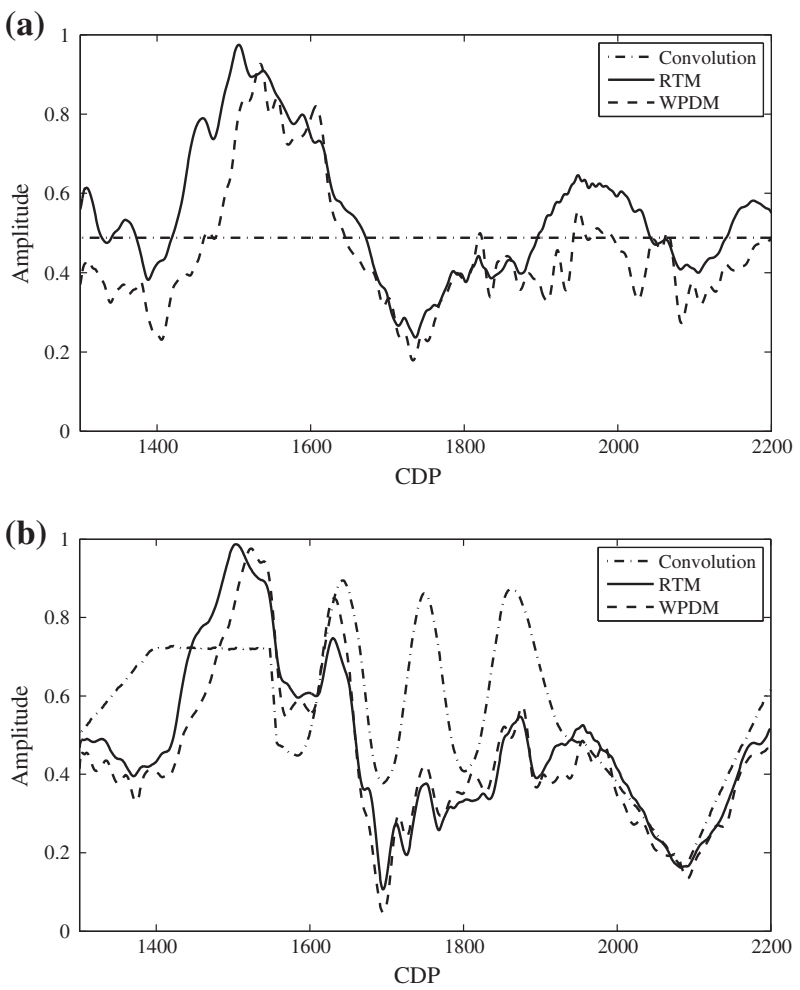

straight line, while curves from WPDM and RTM have seriously deviation (Fig. 7a). The differences between these migrated results and the convolution record come from two sources: the influence of overlying strata and migration method itself. However, the amplitude curves of WPDM and RTM at the first homogeneous layer (H1) shown in Fig. 7 has a high consistency trend. It indicates the amplitude distortion caused by overlying strata is more than migration method. Since reservoir prediction relies on relative amplitude preservation, we ignore the influence of the same overlying strata and compare the curves of WPDM and RTM at H2 layer (Fig. 7b). The two curves have the same shape, which indicates that the two migration methods are equally capable of maintaining seismic reflection characteristics. In other word, RTM has no obvious advantages over WPDM in amplitude preserving for this geologic model.

\section{Velocity model effect}

Velocity model is very important to the migration (Etgen et al. 2009). Conventional, the migrated velocity model in depth domain is always established through velocity analysis, time-depth conversion and tomography. Each step may bring some errors to the final velocity model (Etgen et al. 2009). The errors of velocity may make seriously trouble for the migration. For more realistic evaluation, we process the simulated pre-stack seismic record to obtain the velocity model shown in Fig. 8, and then consider the influence of velocity model. Comparing with the velocity model in Figs. 2 and 8, we can see the outline of salt domes is 


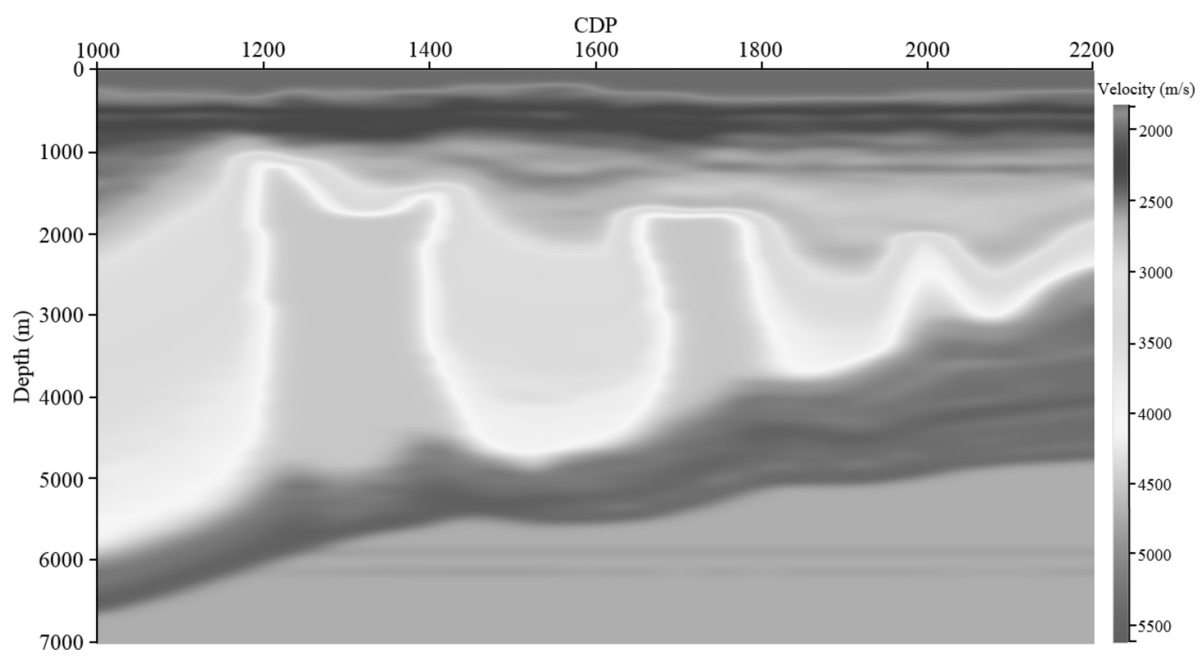

Fig. 8 The analyzed velocity model

clearly but the details and pre-salt structures are smeared in the analyzed velocity model. We also utilize WPDM and RTM operation to the simulated shot record and then obtain the migration results which are shown in Fig. 9a, b. For the salt structure, two methods can roughly depict their shape. In contrast, the salt dome flank and high dip angled formations along the right side of Salt Dome 2 have more accurately image in RTM result than WPDM result. However, for the pre-salt strata, no matter in the image of RTM or in the profile of WPDM, both the reflection characteristics of the organic reef and oolitic beach are difficult to identify. Comparing the migration results in Figs. 4 and 9, we can see the imaging capability by using the above velocity model turns out to be poor. It demonstrates that velocity model has a greater impact on imaging quality than migration method itself.

Likewise, taking the event marked by blue arrow in convolution record (Fig. 5) as a reference, we pick the depth value at the same event in Fig. 9, and then calculate the depth difference between the picked events and the reference event. Compared with the depth error curves of the imaging results in Fig. 10, we can see a significant depth error appear, but the two error curves have a little difference. This demonstrates velocity model has more influence on the imaging quality than the migration method. In addition, ignoring the influence of overlying strata, there is a high consistency between amplitude variation curves of WPDM and RTM results at H2 shown in Fig. 11. It verifies that two migration methods are equally capable of preserving seismic amplitude.

\section{Discussion and conclusion}

According to the actual geologic and reservoir characteristics, the 2D typical complex saltrelated structure geologic model of Pre-Caspian Basin is established. Based on the model, imaging and amplitude-preserving capabilities of WPDM and RTM are compared and analyzed. Some conclusions are drawn as follows:

(1) Both the WPDM and RTM can greatly improve the imaging quality of salt domes but fails in the presence of pre-salt structures. 

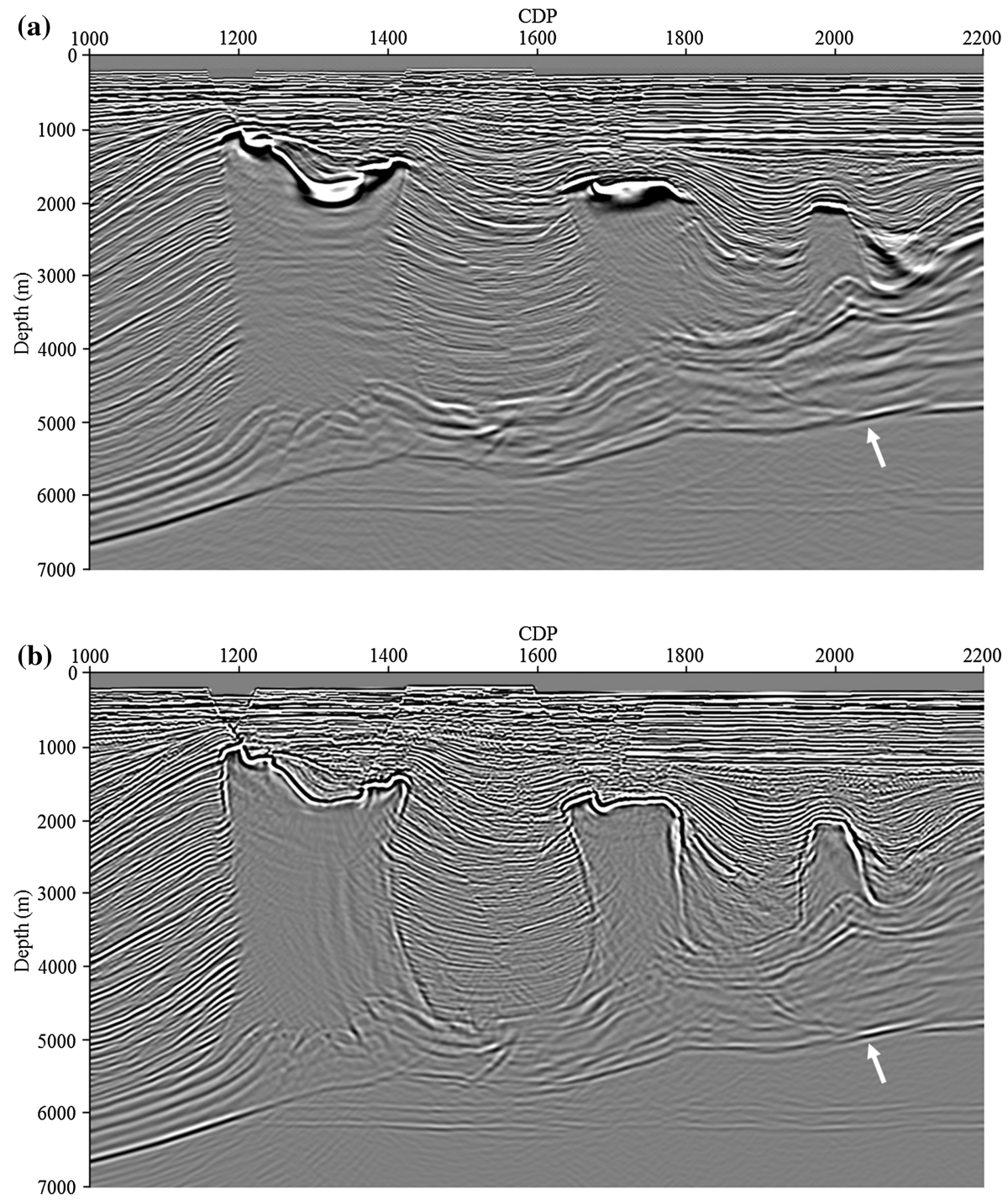

Fig. 9 Comparison between migration profiles of WPDM (a) and RTM (b) using the analyzed velocity model

(2) Because the overburden has strong lateral variation of velocity, RTM and WPDM have not obviously difference on maintaining seismic reflection characteristics of the pre-salt structures. Since amplitude is important for reservoir prediction, the amplitude preservation capability of RTM and WPDM should be improved. The one way is considering the anisotropic behavior into the algorithms. The real underground is anisotropic medium. For example, shale masses overlying dipping salt flanks can be anisotropic, which we currently describe with tilted transverse isotropy (TTI). Therefore, we can adopt acoustic TTI wave equation to make WPDM or RTM operations, which can enhance the seismic imaging and preserve 
Fig. 10 Comparison between imaging depth errors of WPDM result (dash curve) and RTM result (solid curve) at seismic event marked by arrow in Fig. 5 using the analyzed velocity model

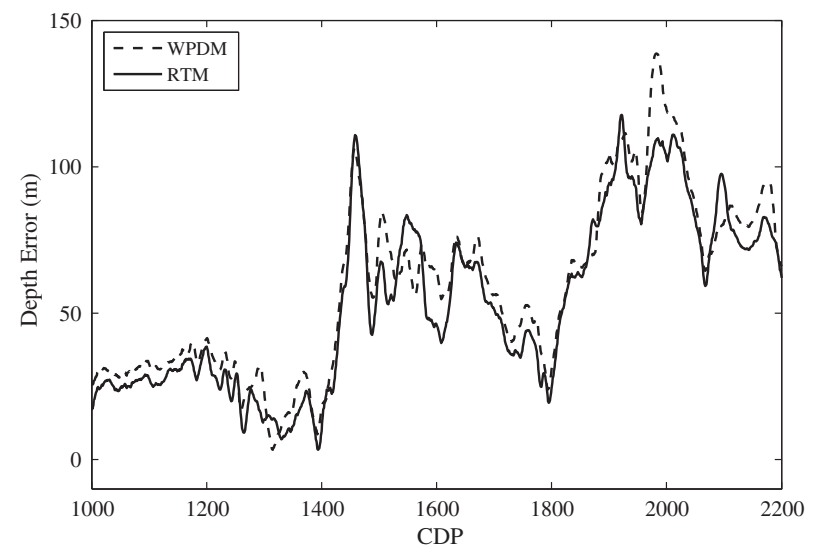

Fig. 11 Comparison between amplitude variation curves at $\mathrm{H} 2$ (Fig. 2) around $6150 \mathrm{~m}$ of WPDM (dash curve) result and RTM (solid curve) result using the analyzed velocity model

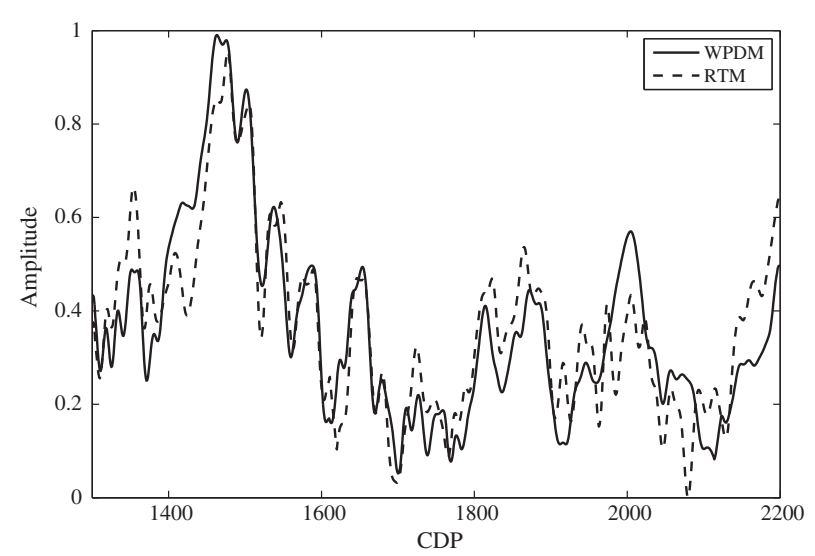

the more realistic amplitude information (Etgen et al. 2009). Another way is improving the amplitude-preserving imaging condition. (Zhang et al. 2007a, b) prove that the 'crosscorrelation' imaging condition is a proper choice to obtain amplitude-preserving angle domain common image gathers (ADCIGs) from a waveequation-based migration in $2 \mathrm{D}$. They re-write the Eq. (7) as,

$$
I(\mathrm{X} ; \theta)=4 \iint \delta\left(\theta^{\prime}\left(x ; x_{s}\right)-\theta\right) \times\left(\int p_{B}\left(x ; t ; x_{s}\right) p_{F}\left(x ; t ; x_{s}\right) d t\right) d \theta^{\prime} d x_{s}
$$

In which, $\theta$ is the reflection angle at the imaging location and the term $\delta\left(\theta^{\prime}\left(x ; x_{s}\right)-\theta\right)$ represents the conversion from shot gathers to reflection angle gathers (Zhang et al. 2014). Therefore, in the future, we suggest adopt the anisotropic method and amplitude-preserving crosscorrelation imaging condition to obtain the ADCIGs and more precisely imaging results.

(3) Velocity model has a greater effect on imaging quality than migration method itself. Therefore, improving the precision of velocity model will play an important role in seismic migration processing. Full wave inversion (FWI) is the latest algorithm to improve the velocity accuracy. Certainly, there is some hope that full waveform 
inversion (FWI) will bring more accurate and detailed models than methods based on moveout. Indeed, many of the difficulties we face in more conventional velocityestimation schemes are because the approximation, the wave-propagation and scattering processing. FWI adopts exactly wave equation to construct velocity model, which reduce the influence from the approximation in seismic processing. Therefore, FWI is one of the best choices to improve the estimation of velocity. Generally, it is not meaning that the other velocity estimation techniques are hopeless. Recently, some methods are proposed by combining conventional gather flatness and wave propagation. Going beyond gather flatness, wave-equation migration velocity analysis methods that rely on focusing of seismic reflection data in the image domain (Weibull and Arntsen 2013; Sava and Biondi 2004; Etgen et al. 2009; Li and Symes 2007) seem to combine the necessary components: kinematics handled by wave propagation and data attributes such as image focusing that are familiar from migration. Additionally, intermediate approaches, i.e., differential semblance ( $\mathrm{Li}$ and Symes 2007), also bridge the gap between gather flatness and waveform inversion. As outgrowths of conventional processing, these two approaches also should be used to improve velocity estimation (Etgen et al. 2009).

Acknowledgments This work is financially supported by Major National Science and Technology Projects (Grant No. 2011ZX05031-001), National Natural Science Foundation of China (Grant No. 41174117) and National Key Basic Research Development Program of China (Grant No. 2013CB228606).

\section{References}

Baysal E, Kosloff DD, Sherwood JWC (1983) Reverse time migration. Geophysics 48(11):1514-1524. doi:10.1190/1.1441434

Bleistein N (1987) On the imaging of reflectors in the earth. Geophysics 52(7):931-942. doi:10.1190/1. 1442363

Etgen J, Gray SH, Zhang Y (2009) An overview of depth imaging in exploration geophysics. Geophysics 74(6):A5-A17. doi:10.1190/1.3223188

Gazdag J (1978) Wave equation migration with the phase-shift method. Geophysics 43(7):1342-1351. doi:10.1190/1.1440899

Leveille JP, Jones IF, Zhou ZZ, Wang B, Liu F (2011) Subsalt imaging for exploration, production, and development: a review. Geophysics 76(5):B3-B20

Li J, Symes WW (2007) Interval velocity estimation via NMO-based differential semblance. Geophysics 72(6):U75-U88. doi:10.1190/1.2767401

Liu F, Zhang G, Morton SA, Leveille JP (2009) An optimized wave equation for seismic modeling and reverse time migration. Geophysics 74(6):A153-A158. doi:10.1190/1.3223678

Liu H, Li B, Tong X, Liu Q, Wang X, Liu W (2012) The issues of prestack reverse time migration and solutions with Graphic Processing Unit implementation. Geophys Prospect 60(5):906-918

Sava P, Biondi B (2004) Wave-equation migration velocity analysis. I. Theory. Geophys Prospect 52(6):593-606. doi:10.1111/j.1365-2478.2004.00447.x

Schneider WA (1978) Integral formulation for migration in two and three dimensions. Geophysics 43(1):49-76. doi:10.1190/1.1440828

Stolt RH (1978) Migration by Fourier transform. Geophysics 43(1):23-48

Sun Y, Qin F, Checkles S, Leveille JP (2000) 3-D prestack Kirchhoff beam migration for depth imaging. Geophysics 65(5):1592-1603. doi:10.1190/1.1444847

Virieux J (1984) SH-wave propagation in heterogeneous media: velocity-stress finite-difference method. Geophysics 49(11):1933-1942. doi:10.1190/1.1441605

Volozh Y, Talbot C, Ismail-Zadeh A (2003) Salt structures and hydrocarbons in the Precaspian Basin. AAPG Bull 87(2):313-334

Weibull WW, Arntsen B (2013) Automatic velocity analysis with reverse-time migration. Geophysics 78(4):S179-S192. doi:10.1190/geo2012-0064.1 
Willis ME, Lu R, Campman X, Toksöz MN, Zhang Y, de Hoop MV (2006) A novel application of timereversed acoustics: salt-dome flank imaging using walkaway VSP surveys. Geophysics 71(2):A7-A11. doi:10.1190/1.2187711

Yuan S, Wang S, Sun W, Miao L, Li Z (2014) Perfectly matched layer on curvilinear grid for the secondorder seismic acoustic wave equation. Explor Geophys 45(2):94-104. doi:10.1071/EG13066

Zhang Y, Xu S, Bleistein N, Zhang G (2007a) True-amplitude, angle-domain, common-image gathers from one-way wave-equation migrations. Geophysics 72(1):S49-S58. doi:10.1190/1.2399371

Zhang Y, Zhang G, Yingst D, Sun J (2007) Explicit marching method for reverse-time migration. SEG technical program expanded abstracts, pp 2300-2304. doi:10.1190/1.2792944

Zhang Y, Ratcliffe A, Roberts G, Duan L (2014) Amplitude-preserving reverse time migration: from reflectivity to velocity and impedance inversion. Geophysics 79(6):S271-S283. doi:10.1190/geo20130460.1 